\title{
Extraocular Sebaceous Carcinoma as a Diagnostic Clue for Muir-Torre
}

\section{Syndrome}

\section{Yazmín Karel Melchor-Mendoza1 ${ }^{1}$ Marcela Saeb-Lima² | Jorge Carlos Salado-Burbano³ | Linda Garcia-Hidalgo ${ }^{1 *}$}

*Correspondence: Linda Garcia-Hidalgo

Address: ${ }^{1}$ Department of Dermatology, Instituto Nacional de Ciencias Médicas y Nutrición Salvador Zubirán. Vasco de Quiroga 15, Belisario Domínguez Secc 16, Tlalpan, 14080, Mexico City, Mexico; ${ }^{2}$ Department of Pathology, Instituto Nacional de Ciencias Médicas y Nutrición Salvador Zubirán. Vasco de Quiroga 15, Belisario Domínguez Secc 16, Tlalpan, 14080, Mexico City, Mexico; ${ }^{3}$ Department of Internal Medicine, The American British Cowdray Medical Center. Sur 136 116, Las Américas, Álvaro Obregón, 01120, Mexico City, Mexico

e-mail $\bowtie$ : lindagh10@yahoo.com

Received: 05 November 2020; Accepted: 16 November 2020

Copyright: (C) 2020 Mendoza YKM. This is an open-access article distributed under the terms of the Creative Commons Attribution License, which permits unrestricted use, distribution, and reproduction in any medium, provided that the original work is properly cited.

\section{ABSTRACT}

A 55-year-old man with a personal and family history of colorectal cancer presented to the dermatology clinic with a 1-month old cutaneous growth on his left buttock. The lesion was subsequently identified as a sebaceous carcinoma, displaying the same mismatch repair gene profile as his previous colorectal carcinoma. A diagnosis of Muir-Torre syndrome was made.

Keywords: Extraocular Sebaceous Carcinoma, Muir-Torre Syndrome

\section{Case Report}

A 55-year-old man presented with a 1-month history of an asymptomatic, rapid growing, yellowish, ulcerated, cupuliform growth on his left buttock (Fig. 1). The patient had been diagnosed with stage IV colorectal adenocarcinoma seven years prior to presentation, with the following mismatch repair (MMR) gene profile: MLH1+, MSH2-, MSH6-, PMS2+. Additionally, his family history was significant for: colorectal cancer in his father and a 28 -year-old nephew, as well as melanoma in his mother.

An excisional skin biopsy was performed (Fig. 2), which reported a moderately differentiated, ulcerated, sebaceous carcinoma (MSH2- and MSH6-). Surgical margins were subsequently enlarged to $1 \mathrm{~cm}$. A diagnosis of Muir-Torre syndrome (MTS) was made based on these findings, as per the 2016 modified diagnostic criteria. 
MTS is an autosomal dominant disease associated with multiple visceral (colorectal, ovarian, urothelial, biliary), and cutaneous (particularly sebaceous) neoplasms. It is considered a variant of Lynch syndrome (John and Schwartz, 2016).

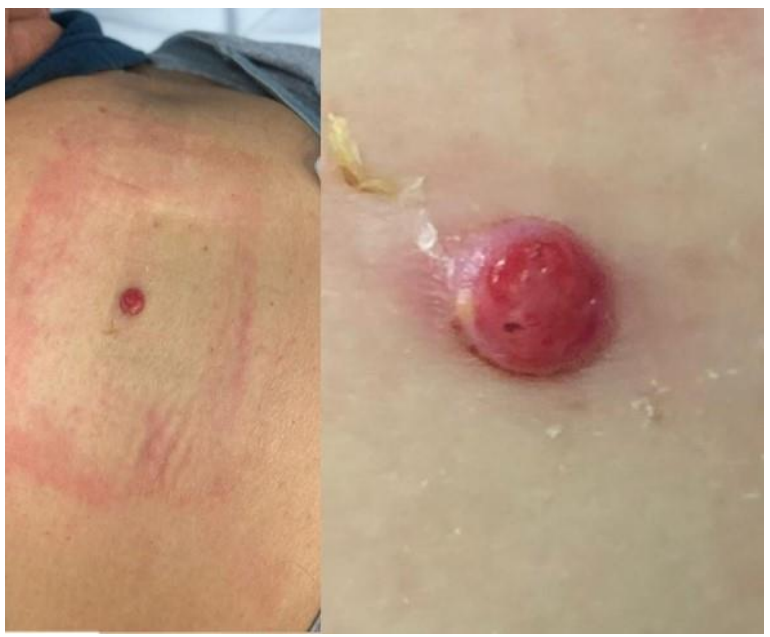

Figure 1: Sebaceous carcinoma located on the left buttock $(1 \mathrm{~cm}$ in diameter). Note the tumor's ulcerated, cupuliform appearance, yellowish-erythematous coloration, well-defined borders, and surrounding collarette of flattened scale

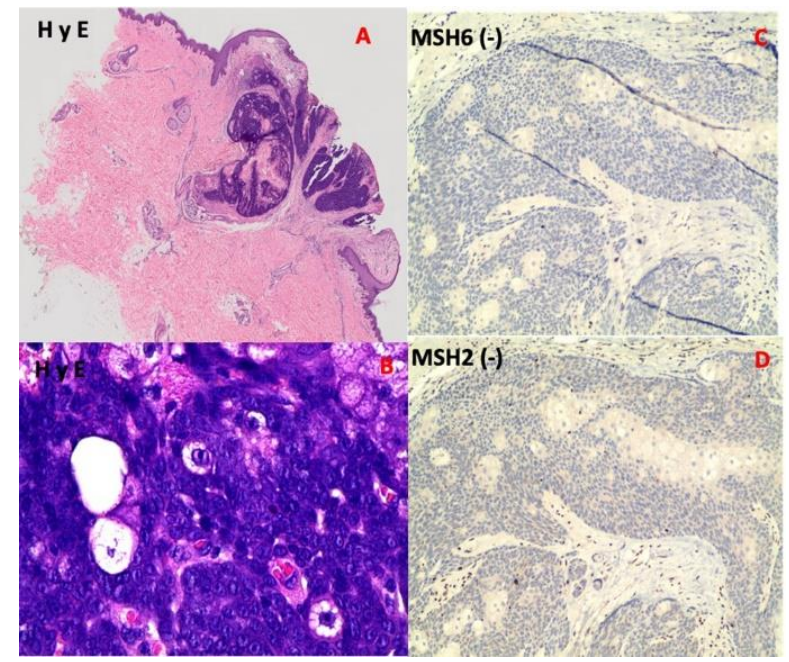

Figure 2: (A) Histopathologic appearance of a moderately differentiated sebaceous carcinoma. (B) Hematoxylin \& Eosin Stain: Dermal neoplasm constituted by sebaceous lobes with areas of focal necrosis and moderately differentiated pleomorphic sebocytes. Immunohistochemical analysis to assess for microsatellite instability showing loss of (C) MSH6 and (D) MSH2 expression

Sebaceous carcinoma (SC) is a rare and aggressive adnexal neoplasm with a high risk of local invasion and metastasis. It can present in an ocular (80\%), or extraocular (20\%) distribution (Chang et al., 2016). Up to $50 \%$ of these lesions are associated with MTS. Extraocular tumors tend to affect the face, neck, or posterior thorax. SC can present with different clinical aspects, either as a uni- or multilobulated, cupuliform growth; or as a yellowish-pink papule. Dermatoscopic examination often 
shows yellow structures, polymorphous vessels, and central ulceration, as seen in our case (Horimoto et al., 2018). SC can precede the diagnosis of visceral malignancy in $56 \%$ of cases, present after the visceral malignancy (22\%), or synchronously (6\%). Histopathological hallmarks include the presence of basaloid cells with sebaceous differentiation, a vacuolated cytoplasm, and indented nuclei. Upon immunohistochemical analysis, neoplastic cells stain positively for epithelial membrane antigen and cytokeratin-7. Regarding microsatellite instability, 63\% are negative for MSH2 and MSH6, 19\% are negative for MLH1 and PMS2, and 19\% are negative for MSH6 (Candelario et al., 2016).

The TNM staging system for cutaneous squamous cell carcinoma is used for this kind of tumor, as there is no specific staging system for extraocular SC. Surgery is the first-line of treatment, with two available modalities. Standard surgical excision with 5-6mm margins offers a local recurrence rate of $36 \%$ within 5 years, and a 30\% 5-year mortality rate. Mohs micrographic surgery offers the advantages of a decreased local recurrence rate (12\% vs 36\%), as well as increased sparing of healthy tissue2. However, it should be noted that the previous data concerns ocular SCs, since data on extraocular SCs is still lacking.

The diagnosis of SC should prompt the clinician to consider the possibility of MTS, particularly in the context of a personal or familial history of visceral malignancy. In the appropriate context, immunohistochemical analysis showing absence of staining for mismatch repair (MMR) genes can be extremely useful to confirm a case of MTS. In our patient's case, both his colorectal adenocarcinoma and sebaceous carcinoma showed absence of MSH2 and MSH6, providing evidence for a common pathophysiological pathway. A high grade of suspicion is necessary to diagnose hereditary cancer syndromes, as their recognition can greatly influence a patient's treatment and surveillance, as well as that of their families.

\section{References}

Candelario NM, Sánchez JE, Sánchez JL, Martín-García RF, Rochet NM. Extraocular Sebaceous Carcinoma - A Clinicopathologic Reassessment. Am J Dermatopathol 2016; 38: 809-812.

Chang AY, Miller CJ, Elenitsas R, Newman JG, Sobanko JF. Management Considerations in Extraocular Sebaceous Carcinoma. Dermatol Surg 2016; 42: S57-S65.

Horimoto K, Kato J, Sumikawa Y, Hida T, Kamiya T, Sato S, Takahashi H, Sawada M, Yamashita T, Uhara H. Dermoscopic features distinctive for extra ocular sebaceous carcinoma. J Dermatol 2018; 45: 487-490.

John AM and Schwartz RA. Muir-Torre syndrome (MTS): An update and approach to diagnosis and management. J Am Acad Dermatol 2016; 74: 558-566. 